Service social

La relation d'aide : éléments de base et guide de perfectionnement ( $3^{e}$ édition), par Jean-Luc Hétu, Boucherville, Gaëtan Morin éditeur, 1990, 181 pages.

\title{
Jean-Marie Boisvert
}

Volume 40, numéro 1, 1991

Éthique et intervention sociale

URI : https://id.erudit.org/iderudit/706521ar

DOI : https://doi.org/10.7202/706521ar

Aller au sommaire du numéro

Éditeur(s)

École de service social de l'Université Laval

ISSN

1708-1734 (numérique)

Découvrir la revue

Citer ce compte rendu

Boisvert, J.-M. (1991). Compte rendu de [La relation d'aide : éléments de base et guide de perfectionnement ( $3^{e}$ édition), par Jean-Luc Hétu, Boucherville, Gaëtan Morin éditeur, 1990, 181 pages.] Service social, 40(1), 146-148.

https://doi.org/10.7202/706521ar d'utilisation que vous pouvez consulter en ligne.

https://apropos.erudit.org/fr/usagers/politique-dutilisation/ 
Puisant dans les écrits et les travaux de recherche sur la prison, l'auteur du livre démontre comment la synergie de complaisance parvient à régulariser les transactions, apaiser la violence potentielle et rendre les rapports quasi harmonieux entre individus aux intérêts si divergents en apparence. Ses réponses sont bien articulées et il maîtrise l'univers qu'il décrit. Avec pertinence, il reconnaît le bien-fondé du mouvement de la démocratie en prison, mais il s'interroge aussi sur les obstacles que la situation risque d'entraîner.

Ce livre, initialement destiné aux intervenants et intervenantes en milieu correctionnel, a le mérite d'analyser le milieu carcéral sans prendre parti pour un des protagonistes. En traçant un portrait fidèle et réaliste de la situation, l'auteur décrit sans juger, expose sans imposer, explique sans endoctriner. II est soucieux de présenter des faits manifestes à son lecteur pour l'aider à comprendre les caractéristiques contraignantes d'un milieu carcéral et les gens qui s'y côtoient : gestionnaires, professionnels, gardiens et prisonniers.

La recommandation d'achat de ce volume convient donc à une grande diversité de personnes, mais particulièrement aux praticiens et praticiennes actuels et futurs des milieux de détention. Il est un ouvrage d'intérêt pour quiconque s'intéresse à la problématique de la criminalité adulte, même sans être directement impliqué dans le milieu.

La structure peu complexe du volume ainsi que le style d'écriture limpide et sans artifice rendent sa lecture facile et agréable. L'argumentation, qui évite les débats idéologiques compliqués, se centre sur l'essentiel et s'appuie sur des données éprouvées par les connaissances et l'expérience. Le fil conducteur de l'ouvrage se retrace logiquement et sans ambiguïté.

Pour terminer, nous formulons deux commentaires moins positifs sur des aspects qui viennent affaiblir quelque peu la qualité de l'ouvrage : une conclusion trop courte qui met fin abruptement à la démarche d'analyse poursuivie et aussi des sources documentaires principales qui commencent à dater. Dans le premier cas, l'auteur donne l'impression d'avoir manqué de souffle pour boucler son ouvrage; dans le second, les références donnent à penser qu'il n'y avait ni plus ni mieux dans la littérature toute récente sur le sujet. Dans les deux cas les impressions auraient pu être évitées compte tenu de la qualité générale du livre et de l'intérêt populaire qu'il pourra susciter.

Germain Trottier

École de service social

Université Laval

\section{La relation d'aide : éléments de base et guide de perfection- nement ( $3^{e}$ éd.), par Jean-Luc HÉTU, Boucherville, Gaëtan Morin, éditeur, 1990, 181 pages.}

Depuis sa première édition en 1982, le livre de Jean-Luc Hétu sur la relation d'aide est devenu une sorte de "classique " dans les universités et collèges du Québec. En 1990, ce manuel en est à sa troisième édition et constituera sans doute encore un outil très utilisé.

Le livre débute par un essai de définition du concept d'aide. L'auteur considère qu'une relation est aidante " lorsqu'elle facilite l'accès aux informations subjectives que sont les sentiments, et qu'elle permet au sujet de clarifier 
ses perceptions " (p. 2). Le rôle de l'aidant consiste alors à écouter activement et à intervenir dans le but de faciliter à l'aidé l'expression, la compréhension et la prise en charge de son vécu. À la suite de cette brève clarification du rôle de l'aidant, l'auteur présente au lecteur un exercice permettant de distinguer différents types d'intervention, d'analyser leur pertinence et d'avoir une première idée de son propre style $d^{\prime}$ 'intervention.

Le modèle de la relation d'aide proposé ici s'inspire très largement de l'approche rogérienne et se base donc essentiellement sur le concept d'empathie, sans toutefois négliger l'apport des connaissances en psychologie et des connaissances professionnelles. Cependant, l'auteur considère qu'il n'existe pas de critères précis permettant de déterminer à quels moments l'aidant doit faire appel à l'empathie, à ses connaissances en psychologie ou à ses connaissances professionnelles. À cet égard, I'aidant doit envisager différentes hypothèses et les vérifier auprès de l'aidé. Le modèle de Jean-Luc Hétu postule également qu'en ce qui concerne les problèmes existentiels (définis ici d'une façon très large), chacun « demeure l'expert de son problème et que c'est lui qui se trouve le mieux placé pour décider en dernière analyse de la solution la plus appropriée pour lui » (p. 43). Il devient alors logique dans un tel modèle de faire peu de place à l'acquisition de compétences personnelles chez l'aidé ou à l'intervention dans l'environnement social.

Après avoir décrit les habiletés nécessaires à la pratique du diagnostic, l'auteur présente les différentes façons d'intervenir. II considère que " l'aidé demande trois choses à son aidant, soit de l'aider à s'exprimer, de l'aider à se comprendre, et de l'aider à changer " et que "l'un de ces besoins est habituellement dominant $》($ p. 106). En accord avec l'approche rogérienne, il favorise généralement l'utilisation du reflet et de la focalisation qui permettraient de passer de l'exploration des sentiments superficiels à celle des sentiments profonds. Mais il propose également d'utiliser la recherche de solutions lorsque l'aidé en est à l'étape du changement. II discute aussi de la place que l'on peut accorder à d'autres façons d'intervenir, telles que l'implication personnelle de l'aidant, l'interprétation et l'exploration des résistances. II accorde toutefois moins de crédit à d'autres méthodes d'intervention telles que la confrontation et les réponses évaluatives du type approbation ou désapprobation de l'aidé, même s'il ne nie pas qu'elles soient parfois utiles, pour autant que "le climat affectif global demeure respectueux et acceptant » (p. 118).

Dans ce livre, Jean-Luc Hétu présente plusieurs notions et plusieurs réflexions qui dénotent une riche expérience clinique. II adopte une approche clairement rogérienne, mais sans rejeter radicalement l'utilisation d'autres modes d'intervention. Toutefois, l'accent est mis sur l'exploration des sentiments et rarement sur l'analyse des comportements, des cognitions ou de l'environnement social. À notre connaissance, il n'existe pas actuellement d'évidence scientifique démontrant qu'il soit préférable de mettre l'accent sur l'une ou l'autre de ces composantes dans toute relation d'aide (ou même dans la majorité des relations d'aide). Cependant, de nombreuses recherches indiquent qu'il est nécessaire de prêter attention à autre chose que les sentiments face à un très grand nombre de problématiques (anxiété, sentiments dépressifs, problèmes conjugaux et familiaux, délinquance, violence et protection de la jeunesse, comportements psychotiques, etc.). On peut donc mettre en doute aujourd'hui l'affirmation que "pour qu'un aidant soit efficace, il suffit qu'il soit empathique, congruent et qu'il manifeste de la considération positive à l'endroit de son aidé " (p. 121). II n'est certes pas possible de relever toutes les méthodes d'aide spécifiques face à des problématiques spécifiques dans un manuel de base sur la relation d'aide. Mais dans une quatrième édition, il 
serait souhaitable de faire une place plus importante à l'analyse des comportements, des cognitions et de l'environnement social.

Le livre de Jean-Luc Hétu s'adresse principalement à des personnes aidantes semi-professionnelles (voir l'avant-propos). Il présente un modèle de type rogérien qui, dans l'état actuel de nos connaissances, ne peut prétendre représenter la seule façon ni même la meilleure façon de conduire une relation d'aide. À la décharge de l'auteur, remarquons que celui-ci discute d'une façon intéressante et souple de quelques méthodes d'intervention d'autres approches (psychanalytique, rationnelle-émotive, etc.). Mais, il s'agit généralement d'approches qui sont aussi centrées sur les émotions plutôt que sur les comportements ou sur l'environnement social. Quoi qu'il en soit, l'apport le plus original de ce livre réside sans doute dans un certain nombre de réflexions importantes, basées sur l'expérience clinique de l'auteur. Notons enfin que l'écriture de Jean-Luc Hétu est claire et précise et que son manuel est bien structuré et facile à consulter.

Jean-Marie Boisvert

École de psychologie

Université Laval

\section{Comprendre pour soigner autrement, par Ellen E. CORIN, Gil- les BIBEAU, Jean-Claude MARTIN et Robert LAPLANTE, Mont- réal, Les Presses de l'Université de Montréal, 1990, 258 pages.}

Dans cet ouvrage les auteurs présentent les résultats d'un travail de recherche conduit sur la question de l'adaptation des services de santé mentale à des réalités locales et régionales. L'approche qu'ils proposent s'inscrit dans un cadre conceptuel et une méthode anthropologique, qui vise à reconstruire le champ des perceptions et des pratiques en santé mentale en prenant en considération le point de vue des communautés. L'hypothèse qui est à la base de leur démarche est " que l'ensemble des perceptions et des représentations relatifs aux problèmes de santé se reflètent directement dans les réactions et dans les actions qu'ils suscitent et influencent notamment la place accordée aux ressources professionnelles» (p. 19).

L'originalité de l'ouvrage est de donner un modèle intéressant de ce qui pourrait être une véritable régionalisation des services de santé en proposant une approche socioculturelle des problèmes de santé mentale. C'est à l'application de cette méthode que nous invitent les auteurs à travers l'étude des problèmes de santé mentale dans trois milieux de l'Abitibi (forestier, agroforestier et minier) qui se sont développés à des périodes historiques différentes.

L'ouvrage comprend six chapitres qui nous entraînent à travers un recadrage anthropologique vers une compréhension des dynamiques locales et des problèmes de santé mentale dans la région de l'Abitibi.

Le premier chapitre nous situe par rapport aux grandes orientations des différents projets de réforme des services québécois de santé, pour ensuite dans le second chapitre nous présenter le contexte social et culturel de la région de l'Abitibi organisé autour de trois grands secteurs économiques primaires : minier, forestier et agro-forestier. Six villes et villages ont été retenus 